\title{
Deeply Virtual Compton Scattering and Generalized Parton Distributions at Jlab/CLAS
}

\author{
Baptiste GUEGAN* \\ IPN Orsay \\ E-mail: guegan@ipno.in2p3.fr
}

The Generalized Parton Distributions (GPDs) provide a new description of nucleon structure in terms of its elementary constituents, the quarks and the gluons. Including and extending the information provided by the form factors and the parton distribution functions, they describe the correlation between the transverse position and the longitudinal momentum fraction of the partons in the nucleon.

Deeply Virtual Compton Scattering (DVCS), the electroproduction of a real photon on a single quark in the nucleon $e N \rightarrow e^{\prime} N^{\prime} \gamma$, is the exclusive process most directly interpretable in terms of GPDs.

A dedicated experiment to study DVCS with the CLAS detector at Jefferson Lab has been carried out using a 5.9-GeV polarized electron beam and an unpolarized hydrogen target, allowing us to collect DVCS events in the widest kinematic range ever explored in the valence region : $1<Q^{2}<$ $4.6 \mathrm{GeV}^{2}, 0.1<x_{B}<0.58$ and $0.09<-t<2 \mathrm{GeV}^{2}$.

In this paper, we show preliminary results of unpolarized cross sections and of polarized cross section differences for the DVCS channel.

Photon 2013,

20-24 May 2013

Paris, France

*Speaker. 


\section{Exclusive process: DVCS}

The Deeply Virtual Compton Scattering (DVCS) provides access to new structure functions, the Generalized Parton Distribution (GPDs), which correlate the spatial parton distribution in the transverse plane with its longitudinal momentum fraction. It is then possible to obtain a three dimensional picture (tomography) of the nucleon.

Approximately fifteen years ago, D. Muller et al. [1], X. Ji [2] and A. Radyushkin [3] showed that in the Bjorken scaling regime the DVCS amplitude factorizes into two parts. One can separate a "hard" part, which consists of the virtual Compton process at the quark level $\left(\gamma^{*} q \rightarrow \gamma q\right)$ that is perturbatively calculable in QED, and a non-perturbative "soft" part, which is parametrized in terms of four GPDs at leading twist and leading order.

Figure. 1 shows the "handbag" diagram for the DVCS process on a nucleon: $e N \rightarrow e^{\prime} N^{\prime} \gamma$. An initial nucleon of momentum $p$ absorbs a virtual photon of momentum $q$ emitted by the incoming electron, producing in the final state an outgoing real photon of momentum $q^{\prime}=q-\Delta$ and a recoil nucleon of momentum $p^{\prime}=p+\Delta$. The quark that absorbs the virtual photon becomes virtual and propagates perturbatively. This quark then radiates a real photon (Compton process) and returns to the nucleon, which is left in its ground state. The quantities $x+\xi(x-\xi)$ of Fig. 1 refer to

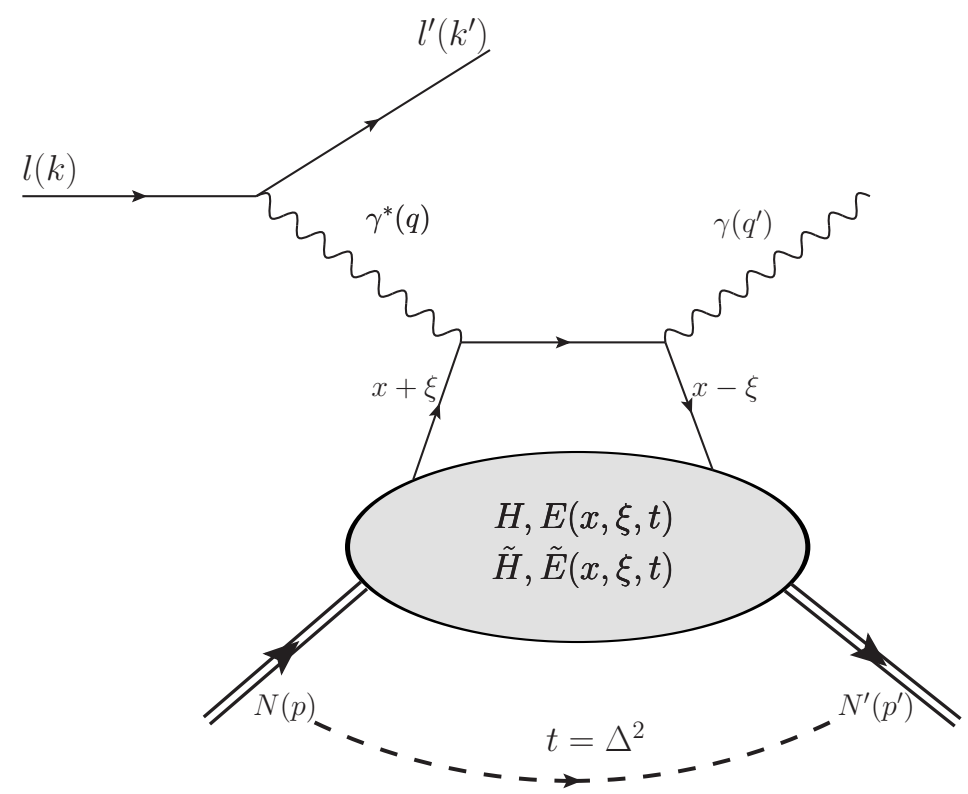

Figure 1: Handbag diagram of the DVCS process in the DGLAP region $x \in[\xi, 1]$, corresponding to the scattering on a quark.

the longitudinal momentum fractions of the initial (final) quark respectively. The variable $x$ is the proton momentum fraction of the struck quark, and $-2 \xi$ is the longitudinal momentum fraction of the transfer $\Delta$. In the Bjorken limit, $\xi$ is related to the standard deep-inelastic Bjorken variable $x_{B}$ via the formula $\xi \sim \frac{x_{B}}{2-x_{B}}$. The variable $t=\Delta^{2}=\left(p^{\prime}-p\right)^{2}$ is the squared momentum transfer 
between the initial and final nucleons.

For each quark flavour $(u, d, s)$, there are four GPDs, $H, E, \tilde{H}, \tilde{E}$, corresponding to each of the four possible helicity-spin configurations of the proton and the quark (Fig. 2). The GPDs $H$ and $E$ are independent on the quark helicity and are therefore called unpolarized GPDs whereas $\tilde{H}$ and $\tilde{E}$ are dependent on the quark helicity and are called polarized GPDs. The GPDs $H$ and $\tilde{H}$ conserve the proton spin, whereas $E$ and $\tilde{E}$ flip the proton spin. In the latter case the overall helicity is not conserved (the proton changes helicity but the quark does not), and angular momentum conservation implies that some orbital angular momentum to be present.

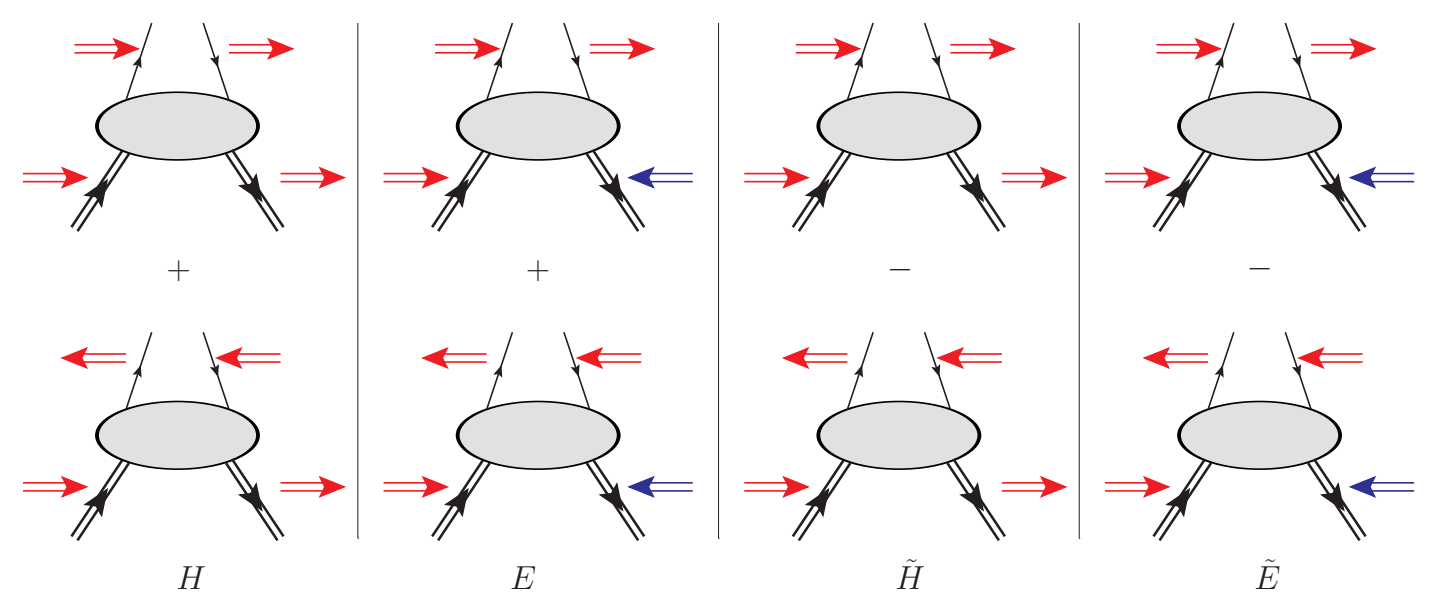

Figure 2: The four nucleon/quark helicity states and associated GPDs. Arrows show the helicity states of the proton and of the quark. Blue arrows indicate the flip of the proton spin. The GPDs $H$ and $E$ are independent of the quark helicity and consist of the linear sum of the proton and quark helicity states. The GPDs $\tilde{H}$ and $\tilde{E}$ depend on the quark helicity and consist of the difference of the proton and quark helicity states.

The DVCS amplitude is complex and can be decomposed as:

$$
\mathscr{M}_{D V C S} \propto \int_{-1}^{1} d x \frac{f(x, \xi, t)}{x \pm \xi \mp i \varepsilon}=\mathscr{P} \int_{-1}^{1} d x \frac{f(x, \xi, t)}{x \pm \xi} \pm i \pi f(x=\mp \xi, \xi, t)
$$

where $f(x, \xi, t)$ stands for any of the four GPDs and $\mathscr{P}$ for the Cauchy principal value integral.

This decomposition leads to the eight quantities presented in Table. 1, called the Compton Form Factors (CFFs) [4]. In this definition, the 8 CFFs are all real functions. They are combinations of weighted GPDs integrated over $x$ and of GPDs evaluated at the line $x= \pm \xi$. They correspond respectively to the real and imaginary parts of the DVCS amplitude, and are the quantities that can actually be extracted from DVCS experiments.

In addition to the DVCS amplitude, the cross section for the exclusive electroproduction of a photon $(e N \rightarrow e N \gamma)$ also receives a contribution from the Bethe-Heitler (BH) process. In this case, 


\begin{tabular}{||c|c||}
\hline Real part & Imaginary part \\
\hline $\mathscr{H}_{R e}(\xi, t)=\mathscr{P} \int_{0}^{1} d x[H(x, \xi, t)-H(-x, \xi, t)] C^{+}(x, \xi)$ & $\mathscr{H}_{I m}(\xi, t)=H(\xi, \xi, t)-H(-\xi, \xi, t)$ \\
\hline $\mathscr{E}_{R e}(\xi, t)=\mathscr{P} \int_{0}^{1} d x[E(x, \xi, t)-E(-x, \xi, t)] C^{+}(x, \xi)$ & $\mathscr{E}_{I m}(\xi, t)=E(\xi, \xi, t)-E(-\xi, \xi, t)$ \\
\hline$\tilde{\mathscr{H}}_{R e}(\xi, t)=\mathscr{P} \int_{0}^{1} d x[\tilde{H}(x, \xi, t)+\tilde{H}(-x, \xi, t)] C^{-}(x, \xi)$ & $\tilde{\mathscr{H}}_{I m}(\xi, t)=\tilde{H}(\xi, \xi, t)+\tilde{H}(-\xi, \xi, t)$ \\
\hline$\tilde{\mathscr{E}}_{R e}(\xi, t)=\mathscr{P} \int_{0}^{1} d x[\tilde{E}(x, \xi, t)+\tilde{E}(-x, \xi, t)] C^{-}(x, \xi)$ & $\tilde{\mathscr{E}}_{I m}(\xi, t)=\tilde{E}(\xi, \xi, t)+\tilde{E}(-\xi, \xi, t)$ \\
\hline
\end{tabular}

Table 1: The eight Compton form factors. The terms corresponding to the real part of the DVCS amplitude are weighted by: $C^{ \pm}(x, \xi)=\frac{1}{x-\xi} \pm \frac{1}{x+\xi}$.

the real photon is emitted by either the incoming or scattered electron, but not by the nucleon itself, unlike for DVCS.

\section{JLab facility}

CEBAF (Continuous Electron Beam Accelerator Facility) is a high intensity, high duty factor electron accelerator whose maximum energy is about $6 \mathrm{GeV}$. CEBAF can deliver electron beams simultaneously to the three experimental halls A, B and C.

In Hall-B, CLAS (CEBAF Large Acceptance Spectrometer)[5] (Figs. 3 and 4) provides identification and kinematics measurements for charged and neutral particles. It has a radius of around 5 meters with a core of six superconducting coils, located around the beamline, that produce a toroidal magnetic field. CLAS has a structure of layered detectors that are split into six symetrical sectors.

CLAS has a large acceptance covering most of the solid angle, making it particularly adapted for the study of exclusive processes. Charged particles can be detected between $5^{\circ}$ and $140^{\circ}$ and neutral particles between $8^{\circ}$ and $45^{\circ}$.

A particle coming from the vertex of the interaction crosses several layers of detectors that track and identificaty it. These detectors consist of:

- 3 layers of drift chambers (DC) that allow the momentum measurement of charged particles via the tracking through the magnetic field

- Cherenkov counters (CC) that distinguish electrons from pions

- scintillators (SC) that provide information for the triggering and the particles' time-of-flight

- electromagnetic calorimeters (EC) that provide the identification and energy measurements of charged and neutral particles in the forward region. 


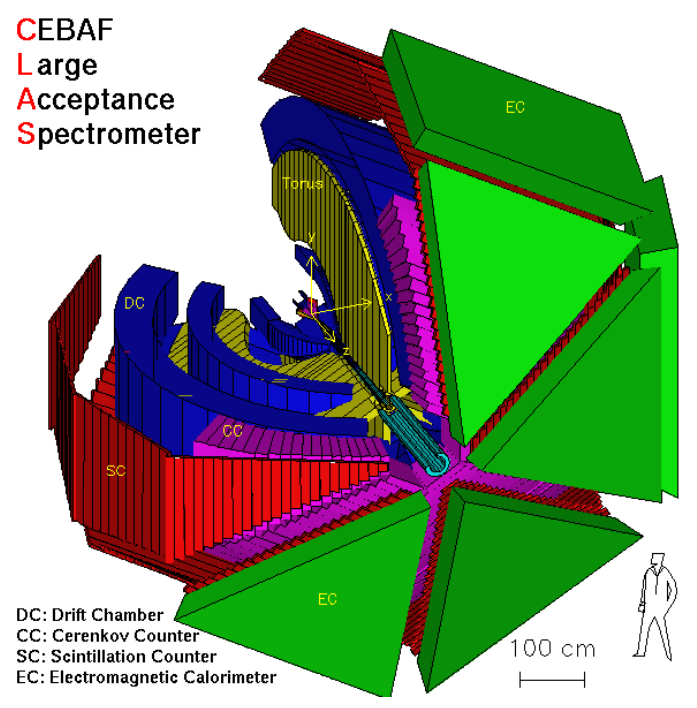

Figure 3: Schematic diagram of CLAS. Four of the six CLAS sectors are displayed, with their different detector layers.

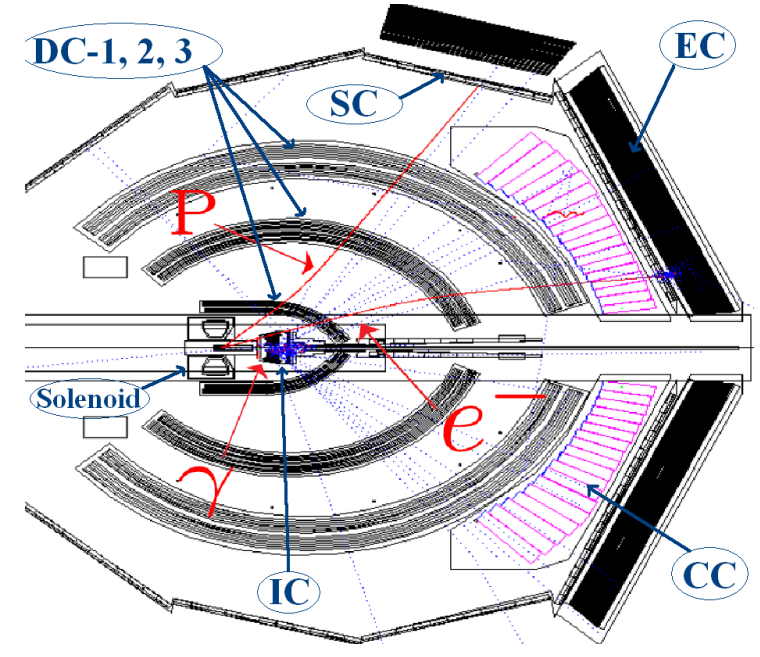

Figure 4: A typical $e p \gamma$ event in a side view of CLAS. The solenoid and the Inner Calorimeter, which are not part of the standard CLAS equipment, can be seen in the central part.

- a new calorimeter specially designed for the DVCS experiment [6], and introduced to cover the forward region at low angle: $5^{\circ} \leq \theta \leq 15^{\circ}$, where DVCS photons are mostly expected to be emitted.

The momentum resolution is of the order of the percent $\left(0.5 \% \leq \frac{\Delta P}{P} \leq 2 \%\right)$ and angular resolutions are $1 \leq \Delta \theta \leq 1.5 \mathrm{mrad}$ and $2 \leq \Delta \phi \leq 3 \mathrm{mrad}$. The time resolution is $100 \leq \Delta t \leq 250$ ps.

\section{Experimental $e p \rightarrow e p \gamma$ cross section}

The data analysis consists of selecting events with one "good" electron, one "good" proton and one "good" photon. To ensure the applicability of the GPD formalism, one requires $Q^{2}>1 \mathrm{GeV}^{2}$ which is meant to select the Bjorken regime, where DVCS factorization should hold. We also require the invariant mass $W=\sqrt{(p+q)^{2}}$ of the hadronic final state to be larger than $2 \mathrm{GeV}$ in order to be above the nucleon resonance region. The phase space $\left(Q^{2}, x_{B}\right)$ thus delimited by these cuts and the acceptance of the detector are shown in Fig. 5. Additional cuts are then applied to ensure the exclusivity of the reaction $e p \rightarrow e p \gamma$.

The data are divided into 21 bins in $\left(Q^{2}, x_{B}\right), 9$ bins in $-t$ and 24 bins in $\Phi$. The 21 bins in $\left(Q^{2}, x_{B}\right)$ are shown in Fig. 5 .

The 4-fold differential DVCS cross section is obtained from:

$$
\frac{d^{4} \sigma}{d Q^{2} d x_{B} d t d \Phi}=\frac{N_{e p \rightarrow e p \gamma}}{A c c L_{i n t} F_{v o l}} \times F_{r a d}
$$

where: 

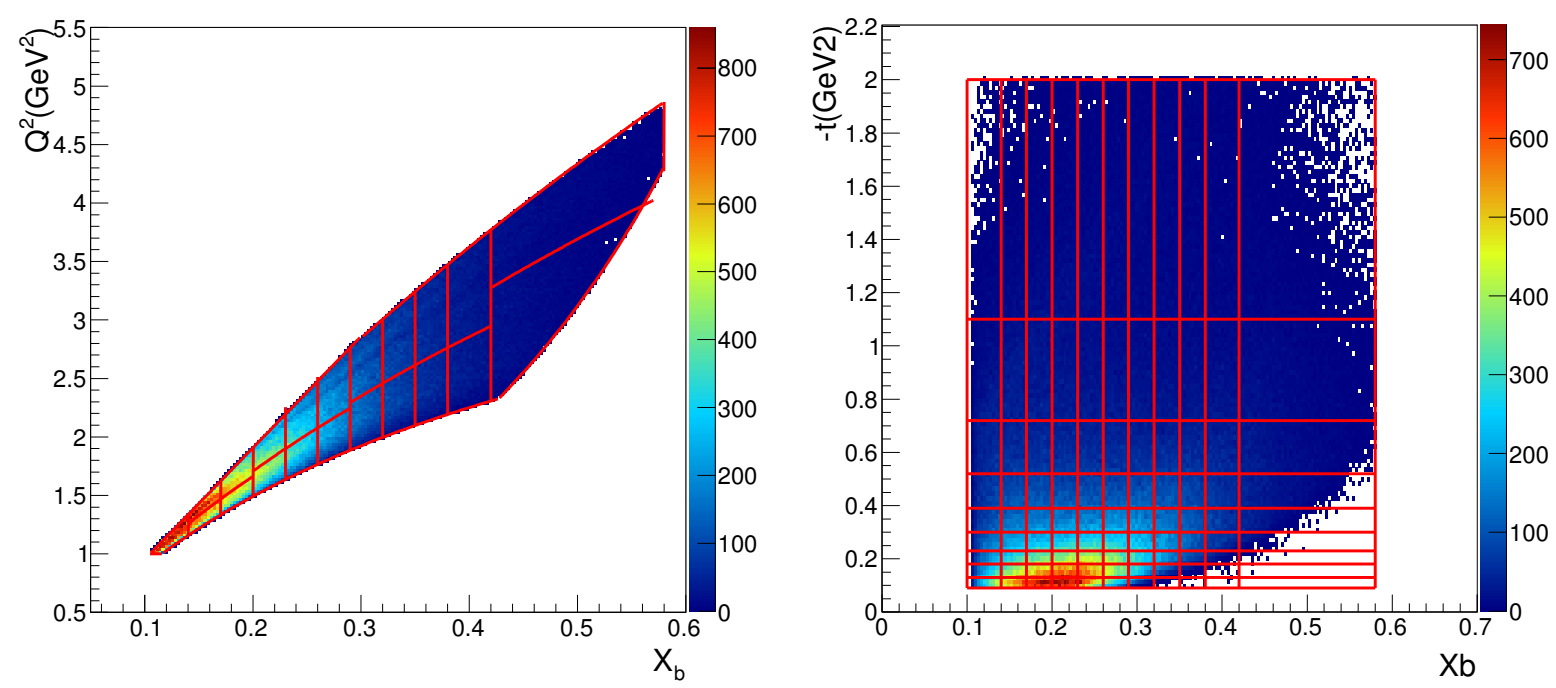

Figure 5: Data binning delimited in red, in $Q^{2}$ vs. $x_{B}$ (left) and $-t$ vs. $x_{B}$ (right).

- $\left(Q^{2}, x_{B}, t, \Phi\right)$ are the four independent variables describing the process.

- $N_{e p \rightarrow e p \gamma}$ is the number of $e p \gamma$ events in each $\left(Q^{2}, x_{B}, t, \Phi\right)$ bins, corrected for $\pi^{0}$ contamination.

- Acc is the acceptance of the detector, determined by simulation for each $\left(Q^{2}, x_{B}, t, \Phi\right)$ bin.

- $L_{\text {int }}$ is the integrated luminosity for the whole DVCS experiment data set.

- $F_{v o l}$ corresponds to the effective hyper-volume of the bin $\left(Q^{2}, x_{B}, t, \Phi\right)$.

- $F_{\text {rad }}$ are the radiative corrections calculated in each $\left(Q^{2}, x_{B}\right)$ bin in order to compute the Born cross section.

In Fig. 6, we present the preliminary unpolarized DVCS cross sections $\frac{d^{4} \sigma}{d Q^{2} d x_{B} d t d \Phi}$ for $\left(Q^{2}, x_{B}\right)$ Bin 2. The total cross-section for the exclusive electroproduction of a photon $(e N \rightarrow e N \gamma)$ receives a contribution from both the DVCS and the BH processes. It is expected that the contribution of the $\mathrm{BH}$ dominates the cross section in the regions where the real photon is emitted in the same direction as the incoming or outgoing electron, which correspond to low and large $\Phi$ regions. The main contribution of the DVCS signal is in the central $\Phi$-region.

For different beam helicity states, the difference of polarized cross sections can be computed for each four-dimensional bin as:

$$
\frac{d^{4} \sigma_{p o l}}{d Q^{2} d x_{B} d t d \Phi}=\frac{1}{2}\left[\frac{d^{4} \vec{\sigma}}{d Q^{2} d x_{B} d t d \Phi}-\frac{d^{4} \overleftarrow{\sigma}}{d Q^{2} d x_{B} d t d \Phi}\right]
$$

In Fig. 7, we show the preliminary results of the polarized cross-sections differences $\sigma_{\text {pol }}$ plotted as a function of $\Phi$ for each of the $9 t$-bins of $\left(Q^{2}, x_{B}\right)$ Bin 2. 

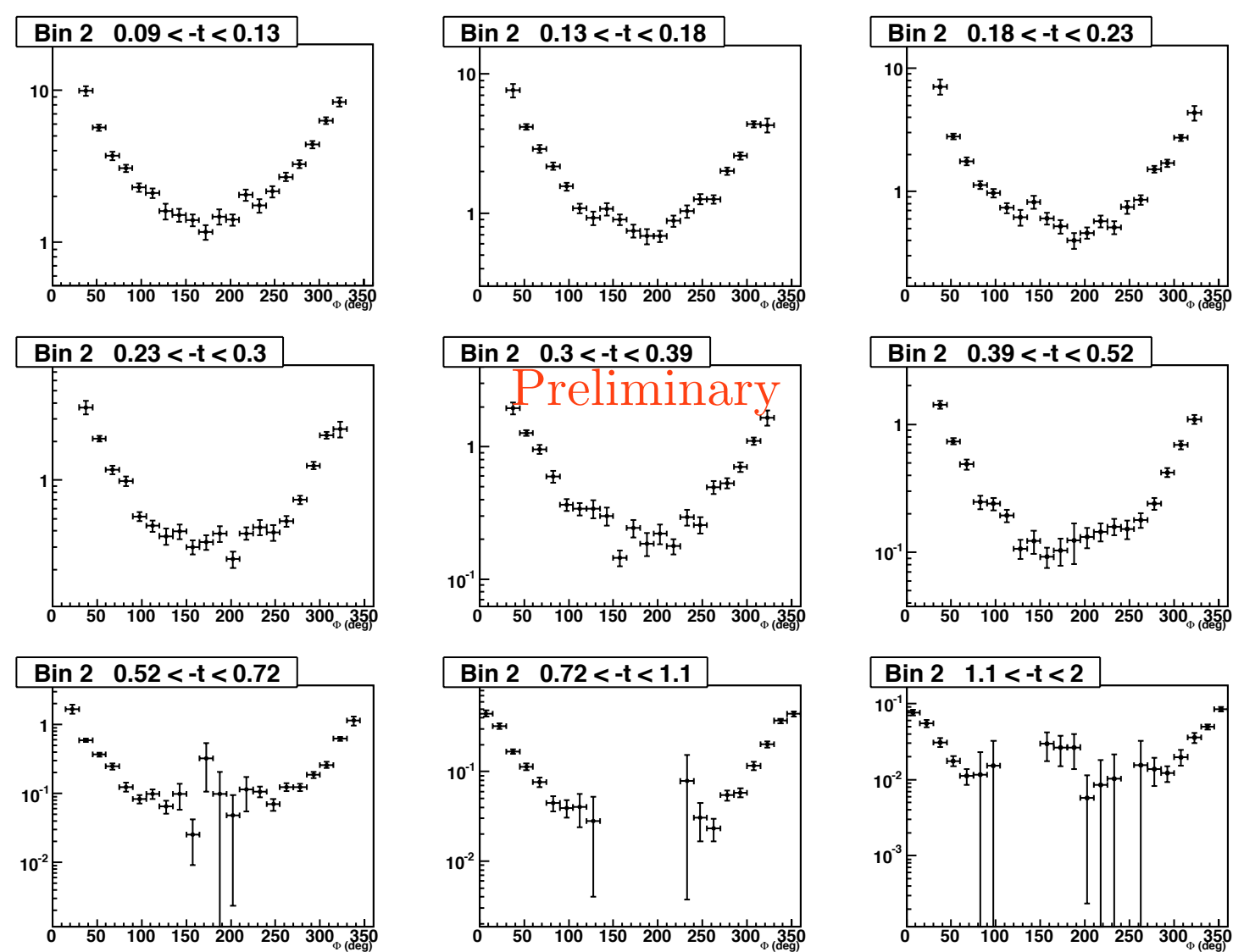

Figure 6: DVCS cross section as a function of $\Phi$ for the $9 t$ bins of $\left(Q^{2}, x_{B}\right)$ Bin 2 . Because these results are still preliminary, we do not display the units on the vertical axis.

\section{Summary}

To conclude, Deeply Virtual Compton Scattering on the proton has been studied in the largest kinematic regime ever explored in the valence region. Using the JLab polarized electron beam operating at $5.88 \mathrm{GeV}$ and an unpolarized hydrogen target, we have extracted the unpolarized cross section and the difference of beam-polarized cross section for the kinematics: $1<Q^{2}<4.6 \mathrm{GeV}^{2}$, $0.1<x_{B}<0.58$ and $0.09<-t<2 \mathrm{GeV}^{2}$. Global fitting of these results will allow the extraction of CFFs, thus providing strong constraints on GPD models over a large kinematic range.

\section{References}

[1] D. Muller, D. Robaschick, B. Geyer, F.M. Dittes and J. Horejsi, Wave Functions, Evolution Equations and Evolution Kernels from Light-Ray Operators of QCD, Fortschr. Phys. 42 (1994) 101.

[2] X. Ji, Gauge-invariant decomposition of nucleon spin and its spin-off, Phys. Rev. Lett. 78 (1997) 610.

[3] A.V. Radyushkin, Scaling limit of Deeply virtual compton scattering, Phys. Lett. B 380 (1996) 417.

[4] M. Guidal, Eur. Phys. J. A 37, (2008) 319 [Erratum-ibid.A40:119, 2009]. 

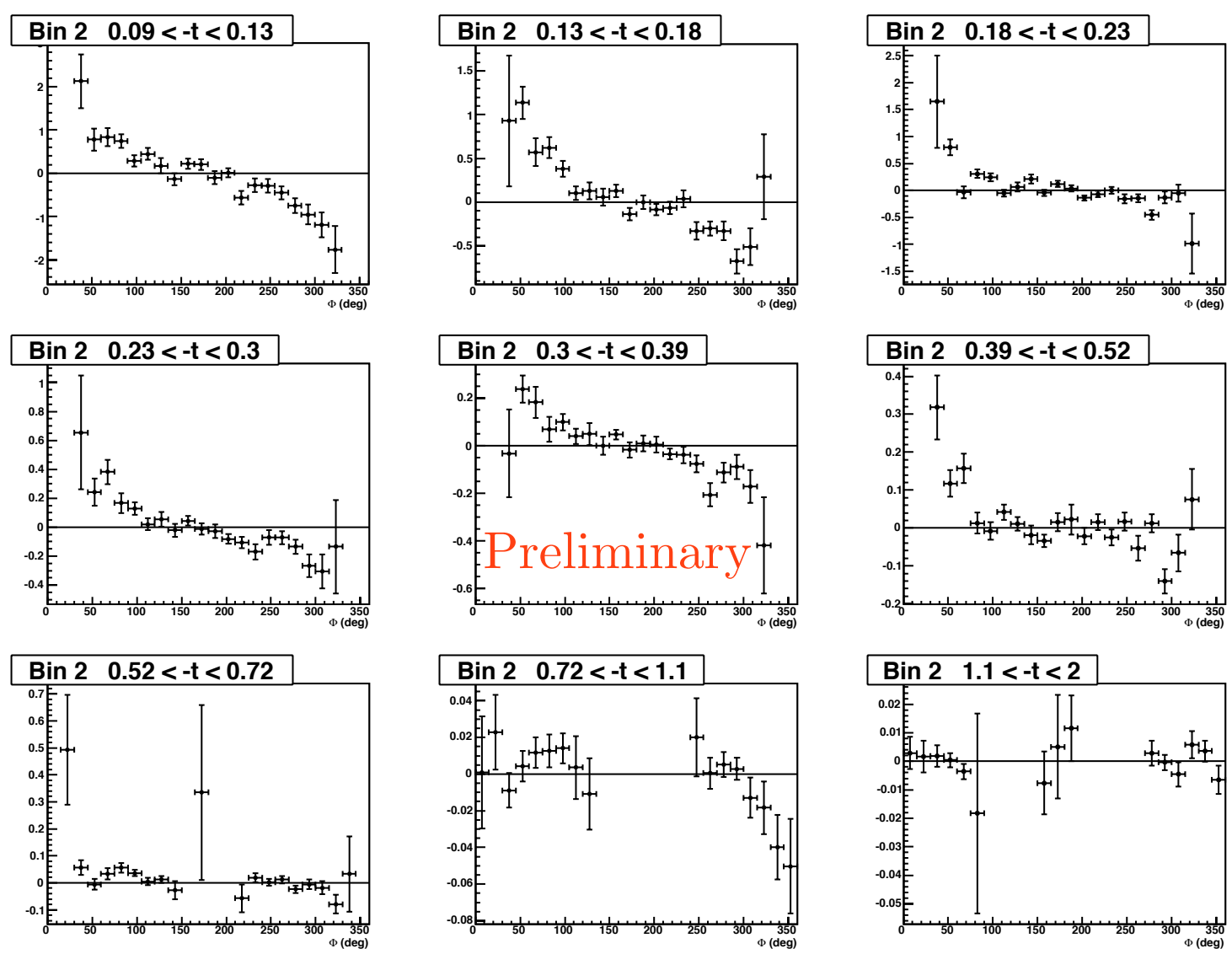

Figure 7: Polarized cross section differences as a function of $\Phi$ for the $9 t$-bins of $\left(Q^{2}, x_{B}\right)$ Bin 2 . Because these results are still preliminary, we do not display the units on the vertical axis.

[5] B.A. Mecking et al., The CEBAF large acceptance spectrometer (CLAS), Nucl. Instrum. Meth. A503 (2003) 513-553.

[6] V. Burkert, L. Elhouadrhiri, M. Garcon, R. Niyazov and S. Stepanyan, Deeply Virtual Compton Scattering with CLAS at $6 \mathrm{GeV}$, JLab approved experiment E-06-003 (2003). 